TRANSACTIONS OF THE

AMERICAN MATHEMATICAL SOCIETY

Volume 361, Number 1, January 2009, Pages 41-59

S 0002-9947(08)04557-1

Article electronically published on August 19, 2008

\title{
ON VISCOSITY SOLUTIONS OF HAMILTON-JACOBI EQUATIONS
}

\author{
SANDRO ZAGATTI
}

\begin{abstract}
We consider the Dirichlet problem for Hamilton-Jacobi equations and prove existence, uniqueness and continuous dependence on boundary data of Lipschitz continuous maximal viscosity solutions.
\end{abstract}

\section{INTRODUCTION}

We consider the Dirichlet problem for Hamilton-Jacobi equations:

$$
\mathcal{P}_{\varphi}: \begin{cases}F(x, u, D u)=0 & \text { in } \Omega \\ u=\varphi & \text { in } \partial \Omega,\end{cases}
$$

where $\Omega$ is an open bounded subset of $\mathbb{R}^{n}, F: \Omega \times \mathbf{R} \times \mathbf{R}^{n} \rightarrow \mathbf{R}$ is a continuous function and $\varphi$ is a boundary datum that is assumed to belong to $W^{1, \infty}(\Omega)$. In this paper by a solution of $\mathcal{P}_{\varphi}$ we mean an element $u$ of the set $\varphi+W_{0}^{1, \infty}(\Omega)$ such that $F(x, u(x), D u(x))=0$ for almost every $x \in \Omega$, and by a viscosity solution we mean an element $u$ of the set $\varphi+W_{0}^{1, \infty}(\Omega)$ solving the equation in the viscosity sense.

The problem of existence of such kinds of solutions has been widely studied by various authors, and we mention the monographs [13, 2], 10] and the papers [1], 3], 4], 6], 7], 9], and [1]. In particular in 9] the authors consider the homogeneous case corresponding to a function $F$ depending only on $D u$ and to $\varphi \equiv$ 0 , providing an explicit formula for a Lipschitz continuous solution, that they call fundamental, which turns out to be a viscosity solution and, under some additional hypotheses, unique.

In this paper we consider the general case, assuming that

$$
\lim _{|\xi| \rightarrow \infty} F(x, u, \xi)=+\infty
$$

uniformly in $(x, u) \in \Omega \times \mathbf{R}$ and that

$$
\{(x, u, \xi): F(x, u, \xi)>0\}=\left\{(x, u, \xi): F^{* *}(x, u, \xi)>0\right\},
$$

where $F^{* *}$ denotes the lower convex envelope of $F$ with respect to the last variable.

Given a boundary datum $\varphi$ such that $F^{* *}(x, \varphi, D \varphi) \leq 0$ almost everywhere in $\Omega$, we prove the existence of a (pointwise) maximal element $\bar{u}$ of the set

$$
S_{\varphi}=\left\{u \in \varphi+W_{0}^{1, \infty}(\Omega): F^{* *}(x, u, D u) \leq 0 \quad \text { a.e. in } \Omega\right\}
$$

Received by the editors August 21, 2006.

2000 Mathematics Subject Classification. Primary 49L25.

(C)2008 American Mathematical Society Reverts to public domain 28 years from publication 
which turns out to be a viscosity solution of $\mathcal{P}_{\varphi}$ and of $\mathcal{P}_{\varphi}^{* *}$. The method that we use is inspired by the results contained in [15, [16, [17] and [18, which are mainly devoted to the minimization of nonsemicontinuous functionals of the Calculus of Variations.

The maximality of $\bar{u}$ implies automatically its uniqueness, and then it is natural to ask if such a solution depends continuously on the boundary data. This problem is treated in section 4 where it is shown that if the boundary of $\Omega$ is Lipschitz and $\varphi, \psi$ are two boundary data close to each other in the uniform norm on $\partial \Omega$, then the corresponding maximal solutions provided by Theorem 2 are close to each other in the weak* topology of $W^{1, \infty}(\Omega)$.

In addition we give simple examples showing that our hypotheses, in general, cannot be weakened and, in section 5, we discuss briefly some consequences of our result in the special homogeneous case.

\section{Preliminaries and notation}

In this paper $\mathbb{R}^{n}$ is the euclidean $n$-dimensional space, and we denote respectively by $\langle\cdot, \cdot\rangle$ and by $|\cdot|$ the inner product and the euclidean norm in it. For $x \in \mathbf{R}^{n}$ and $r>0, B(x, r)$ is the open ball in $\mathbf{R}^{n}$ of center $x$ and radius $r$. Given $E \subseteq \mathbb{R}^{n}$, meas $(E)$ is the Lebesgue measure, $\partial E$ is the boundary, $E^{c}$ is the complement and $\operatorname{co}(E)$ is the convex hull of $E$; $\operatorname{extr}(E)$ is the set of extremal points of a convex set $E$. By dist $(x, E)$ we denote the distance of the point $x$ from the set $E$ and by $\operatorname{dist}(A, B)$ the distance between two subsets of $\mathbf{R}^{n}$.

Throughout the paper $\Omega$ is an open bounded subset of $\mathbf{I R}^{n}$; we use the spaces $C^{k}(\Omega), C_{c}^{k}(\Omega), L^{p}(\Omega), W^{1, p}(\Omega)$ and $W_{0}^{1, p}(\Omega)$, for $k \in \mathbb{I}_{0}=\{0\} \cup \mathbb{I N}$ and $1 \leq$ $p \leq \infty$, with their usual (strong and weak) topologies. By $\operatorname{supp}(v)$ we denote the support of the function $v$ and, dealing with Sobolev functions, we assume to use the precise representative as defined, for example, in [12.

We need the following notion and refer to the monograph [2] (chapter II) for proofs, general setting and for the definition of viscosity solutions of HamiltonJacobi equations.

Definition 1. Let $U \subseteq \mathbb{R}^{n}$ be open, $v \in C^{0}(U)$ and $x_{0} \in U$. We set

$$
\begin{aligned}
D^{-} v\left(x_{0}\right) & \doteq\left\{\xi \in \mathbb{R}^{n}: \liminf _{x \rightarrow x_{0}} \frac{v(x)-v\left(x_{0}\right)-\left\langle\xi, x-x_{0}\right\rangle}{\left|x-x_{0}\right|} \geq 0\right\}, \\
D^{+} v\left(x_{0}\right) & \doteq\left\{\xi \in \mathbb{R}^{n}: \limsup _{x \rightarrow x_{0}} \frac{v(x)-v\left(x_{0}\right)-\left\langle\xi, x-x_{0}\right\rangle}{\left|x-x_{0}\right|} \leq 0\right\} .
\end{aligned}
$$

We call these sets, respectively, super and sub differentials (or semidifferentials) of $v$ at the point $x_{0}$ and set also

$$
\begin{aligned}
& A^{-}(v) \doteq\left\{x \in U: D^{-} v(x) \neq \emptyset\right\}, \\
& A^{+}(v) \doteq\left\{x \in U: D^{+} v(x) \neq \emptyset\right\} .
\end{aligned}
$$

We recall the following.

Lemma 1. Let $U \subseteq \mathbb{R}^{n}$ be open, $v \in C^{0}(U)$ and $x_{0} \in U$.

(i) $\xi \in D^{-} v\left(x_{0}\right)$ if and only if there exists a function $\phi \in C^{1}(U)$ such that $D \phi\left(x_{0}\right)=\xi$ and the function $x \mapsto v(x)-\phi(x)$ has a local minimum at the point $x_{0}$. 
(ii) $\xi \in D^{+} v\left(x_{0}\right)$ if and only if there exists a function $\phi \in C^{1}(U)$ such that $D \phi\left(x_{0}\right)=\xi$ and the function $x \mapsto v(x)-\phi(x)$ has a local maximum at the point $x_{0}$.

(iii) $D^{+} v\left(x_{0}\right)$ and $D^{-} v\left(x_{0}\right)$ are closed convex possibly empty subsets of $\mathbf{R}^{n}$.

(iv) If $v$ is differentiable at the point $x_{0}$, then $D^{+} v\left(x_{0}\right)=D^{-} v\left(x_{0}\right)=\left\{D v\left(x_{0}\right)\right\}$.

(v) If both $D^{+} v\left(x_{0}\right)$ and $D^{-} v\left(x_{0}\right)$ are nonempty then $u$ is differentiable at $x_{0}$ and $D^{+} v\left(x_{0}\right)=D^{-} v\left(x_{0}\right)=\left\{D v\left(x_{0}\right)\right\}$.

(vi) The sets $A^{-}(v)$ and $A^{+}(v)$ are dense in $U$.

Remark 1. The proof of the above lemma can be found in [2] and we take from such a textbook the following argument (Lemma 1.7, p. 29), which is a direct consequence of definitions (2.1), (2.2) and of Lemma 1.

(i) Let $\xi \in D^{-} v\left(x_{0}\right)$. Then there exist $\rho>0$ and a continuous increasing function $\sigma:[0,+\infty[\rightarrow[0,+\infty[$ such that $\sigma(0)=0$ and

$$
v(x) \geq v\left(x_{0}\right)+\left\langle\xi, x-x_{0}\right\rangle-\sigma\left(\left|x-x_{0}\right|\right)\left|x-x_{0}\right| \quad \forall x \in B\left(x_{0}, \rho\right) .
$$

(ii) Let $\xi \in D^{+} v\left(x_{0}\right)$. Then there exist $\rho>0$ and a continuous increasing function $\sigma:[0,+\infty[\rightarrow[0,+\infty[$ such that $\sigma(0)=0$ and

$$
v(x) \leq v\left(x_{0}\right)+\left\langle\xi, x-x_{0}\right\rangle+\sigma\left(\left|x-x_{0}\right|\right)\left|x-x_{0}\right| \quad \forall x \in B\left(x_{0}, \rho\right) .
$$

In the proof of our results we shall need the following refined version of a wellknown argument (see [11, [16, 17]) in which we make use of Stampacchia's theorem (see, for example, theorem 4, page 129 of [12]) according to which the pointwise maximum $w \doteq u \wedge v$ of two Sobolev functions $u$ and $v$ is still a Sobolev function whose gradient $D w$ equals almost everywhere the gradients $D u$ or $D v$, according to the value taken by the function $w$.

Lemma 2. Let $U$ be an open subset of $\mathbb{R}^{n}, p \in[1, \infty], v \in W^{1, p}(U, \mathbb{R}) \cap C^{0}(U, \mathbb{R})$, $x_{0} \in A^{-}\left(x_{0}\right), \xi \in D^{-} v\left(x_{0}\right), r>0, t>0$ and $\rho>0$ such that $B\left(x_{0}, \rho\right) \subseteq U$. Then there exists a map $\hat{v} \in W^{1, p}(U, \mathbf{R}) \cap C^{0}(U, \mathbf{R})$ with the following properties:

$$
\begin{aligned}
& \hat{v}-v \in W_{0}^{1, p}(U) ; \\
& v(x) \leq \hat{v}(x) \leq v(x)+t \text { for a.e. } x \in U ; \\
& \hat{\Lambda} \doteq\{x \in U: \hat{v}(x)>v(x)\} \text { is nonempty and } \hat{\Lambda} \subseteq B\left(x_{0}, \rho\right) ; \\
& \left\{\begin{array}{c}
|D \hat{v}(x)-\xi|=r, \text { for a.e. } x \in \hat{\Lambda} \\
D \hat{v}(x)=D v(x), \text { for a.e. } x \in U \backslash \hat{\Lambda} ;
\end{array}\right. \\
& \int_{U} \hat{v} d x>\int_{U} v d x .
\end{aligned}
$$

Proof. Reducing if necessary $\rho$ we may assume that

$$
\rho \leq \frac{t}{3(r+|\xi|)}
$$

and that

$$
\left|v(x)-v\left(x_{0}\right)\right| \leq \frac{t}{3} \quad \forall x \in \overline{B\left(x_{0}, \rho\right)},
$$

where the last inequality follows from uniform continuity of $v$ on compact subsets of $U$.

Take $s>0$ satisfying

$$
s \leq \min \{t, r \rho\}
$$


and such that, recalling (2.3),

$$
\frac{v(x)-v\left(x_{0}\right)-\left\langle\xi, x-x_{0}\right\rangle}{\left|x-x_{0}\right|} \geq-\frac{r}{2} \quad \forall x \in B\left(x_{0}, \frac{s}{r}\right) .
$$

By the choice (2.11), (2.12) implies, in particular:

$$
v(x)-v\left(x_{0}\right)-\left\langle\xi, x-x_{0}\right\rangle \geq-\frac{s}{2} \quad \forall x \in B\left(x_{0}, \frac{s}{r}\right) .
$$

Define a map $w$ on $B\left(x_{0}, \frac{s}{r}\right)$ by setting

$$
w(x) \doteq \max \left\{v(x), v\left(x_{0}\right)+\left\langle\xi, x-x_{0}\right\rangle+\frac{s}{4}-r\left|x-x_{0}\right|\right\}
$$

and, first of all, notice that by (2.9), (2.10) and (2.11), we have

$$
|w(x)-v(x)| \leq\left|v(x)-v\left(x_{0}\right)\right|+(|\xi|+r) \rho+\frac{s}{4} \leq t .
$$

Introduce the set

$$
\hat{\Lambda} \doteq\left\{x \in B\left(x_{0}, \frac{s}{r}\right): w(x)>v(x)\right\} .
$$

Since both the functions $v$ and

$$
B\left(x_{0}, \rho\right) \ni x \mapsto v\left(x_{0}\right)+\left\langle\xi, x-x_{0}\right\rangle+\frac{s}{4}-r\left|x-x_{0}\right|
$$

are continuous and the value of the second one in $x_{0}$ is strictly greater than $v\left(x_{0}\right)$, it follows that the set $\hat{\Lambda}$ is nonempty. In addition the map defined in (2.17) is Lipschitz continuous and then belongs to $W^{1, \infty}\left(B\left(x_{0}, \frac{s}{r}\right)\right)$, which is contained in $W^{1, p}\left(B\left(x_{0}, \frac{s}{r}\right)\right)$; hence, by Stampacchia's theorem and by the continuity of $v$ and of the map in (2.17), $w$ belongs to $W^{1, p}\left(B\left(x_{0}, \frac{s}{r}\right)\right) \cap C^{0}\left(B\left(x_{0}, \frac{s}{r}\right)\right)$ and we have:

$$
D w(x)= \begin{cases}\xi-r D\left|x-x_{0}\right|, & \text { for a.e. } x \in \hat{\Lambda}, \\ D v(x), & \text { for a.e. } x \in B\left(x_{0}, \frac{s}{r}\right) \backslash \hat{\Lambda} .\end{cases}
$$

We observe that, for any $x \in B\left(x_{0}, \frac{s}{r}\right)$ such that $\left|x-x_{0}\right|>\frac{3}{4} \frac{s}{r}$, we have, by (2.13),

$$
\begin{aligned}
& v\left(x_{0}\right)+\left\langle\xi, x-x_{0}\right\rangle+\frac{s}{4}-r\left|x-x_{0}\right| \\
& \quad<v\left(x_{0}\right)+\left\langle\xi, x-x_{0}\right\rangle+\frac{s}{4}-\frac{3}{4} s \\
& \quad=v\left(x_{0}\right)+\left\langle\xi, x-x_{0}\right\rangle-\frac{s}{2} \\
& \quad \leq v(x) .
\end{aligned}
$$

Hence

$$
\hat{\Lambda} \subseteq B\left(x_{0}, \frac{s}{r}\right)
$$

and the map $w$ coincides with $v$ on $B\left(x_{0}, \frac{s}{r}\right) \backslash \overline{B\left(x_{0}, \frac{3}{4} \frac{s}{r}\right)}$. By definition (2.14) and by standard notions on Sobolev functions (see for example [12]), this implies that

$$
\left.(w-v)\right|_{B\left(x_{0}, \frac{s}{r}\right)} \in W_{0}^{1, p}\left(B\left(x_{0}, \frac{s}{r}\right)\right) .
$$

Now we set

$$
\hat{v}(x) \doteq \begin{cases}w(x) & \text { for } x \in B\left(x_{0}, \frac{s}{r}\right) \\ v(x) & \text { for } x \in U \backslash B\left(x_{0}, \frac{s}{r}\right)\end{cases}
$$


From (2.14), (2.19) (and the consideration which follows), (2.20) and (2.21) we obtain that $\hat{v}$ lies in $W^{1, p}(U) \cap C^{0}(U)$. We see now that conditions (2.4)-(2.8) hold. Property (2.4) is a trivial consequence of (2.14), (2.20) and (2.21); (2.5) follows trivially from definitions (2.14), (2.21) and from (2.15). Property (2.6) follows directly from definition (2.16) and, in order to show (2.7), we observe that, by (2.18) and by (2.21), we have that $|D \hat{v}(x)-\xi|=r|D| x-x_{0}||=r$ for almost every $x \in \hat{\Lambda}$ and that $D \hat{v}(x)=D v(x)$ for almost every $x \in U \backslash \hat{\Lambda}$. Inequality (2.8) is a consequence of (2.5) and of the fact that the open set $\hat{\Lambda}$, being nonempty, has positive measure.

Remark 2 . We shall use Lemma 1 only in the case $p=\infty$ so that the continuity of $v$ is automatically guaranteed.

The following lemma completes an argument introduced in [17] for maps belonging to $W^{1, p}(\Omega)$ and $1 \leq p<\infty$.

Lemma 3. Let $\Omega$ be an open bounded subset of $\mathbf{R}^{n}$ with Lipschitz boundary $\partial \Omega$. Let $t \in \mathbb{R}^{+}$be small and $u, v \in W^{1, \infty}(\Omega)$ be such that

$$
\|u\|_{W^{1, \infty}(\Omega)},\|v\|_{W^{1, \infty}(\Omega)} \leq M
$$

and

$$
\|u-v\|_{C(\partial \Omega)} \leq t
$$

Then there exist an open subset $\Omega_{t} \subseteq \Omega$, a map $w_{t} \in W^{1, \infty}(\Omega)$ and a continuous increasing function $\sigma:[0,+\infty[\rightarrow[0,+\infty[$, depending only on $M$ and on $\Omega$, with $\sigma(0)=0$ such that

$$
\begin{aligned}
& \Omega_{t} \subseteq \Omega_{s} \quad \text { if } t>s ; \\
& \operatorname{meas}\left(\Omega \backslash \Omega_{t}\right) \rightarrow 0 \quad \text { as } \quad t \rightarrow 0+ \\
& w_{t}=u \quad \text { in } \Omega_{t} ; \\
& w_{t}=v \quad \text { in } \quad \partial \Omega \\
& \left|w_{t}-u\right|,\left|w_{t}-v\right| \leq \sigma(t) \quad \text { in } \quad \Omega \backslash \Omega_{t} ; \\
& \operatorname{dist}\left(D w_{t}(x), \operatorname{co}(\{D u(x), D v(x)\})\right) \leq \sigma(t) \quad \text { for a.e. } \quad x \in \Omega .
\end{aligned}
$$

Proof. Step 1. We first assume $u \equiv 0$ so that condition (2.23) takes the form

$$
\|v\|_{C(\partial \Omega)} \leq t
$$

We localize the problem by considering a point $x \in \partial \Omega$ and $r>0$ such that, up to an orthogonal change of variables and for a suitable Lipschitz continuous function $\gamma: \mathbb{R}^{n-1} \rightarrow \mathbb{R}$, we have:

$$
\Omega \cap Q(x, r)=\left\{y=\left(y^{\prime}, y_{n}\right): y_{n}>\gamma\left(y^{\prime}\right)\right\} \cap Q(x, r),
$$

where we adopt the notation $y=\left(y_{1}, \ldots, y_{n-1}, y_{n}\right)=\left(y^{\prime}, y_{n}\right)$, and $Q(x, r)$ denotes the cube

$$
Q=Q(x, r) \doteq\left\{y=\left(y_{1}, \ldots, y_{n}\right):\left|y_{i}-x_{i}\right|<r, i=1, \ldots, n\right\} .
$$

We set also

$$
\Delta(x, r) \doteq\left\{y^{\prime}=\left(y_{1}, \ldots, y_{n-1}\right):\left|y_{i}-x_{i}\right| \leq r, i=1, \ldots, n-1\right\} .
$$

Step 2. Assume that the support of $v$ is contained in $\bar{\Omega} \cap Q$; set

$$
Z \doteq(\bar{\Omega} \cap Q) \backslash \operatorname{supp}(v)
$$


and notice that (2.22) and (2.30) imply that

$$
|v(x)| \leq t+M s \quad \forall x \in \Omega: \operatorname{dist}(x, \partial \Omega) \leq s .
$$

Since we are interested in small values of the parameter $t$, we may assume that

$$
\left\{y=\left(y^{\prime}, y_{n-1}\right): y^{\prime} \in \Delta(x, r), \gamma\left(y^{\prime}\right) \leq y_{n} \leq \gamma\left(y^{\prime}\right)+t^{\frac{1}{2}}\right\} \subseteq Q(x, r) \cap \bar{\Omega} .
$$

Define the maps $I^{ \pm}: Q(x, r) \cap \bar{\Omega} \rightarrow \mathbb{R}$ by setting

$$
\begin{array}{ll}
I^{+}\left(y^{\prime}, y_{n}\right)=t-t^{\frac{1}{2}}\left(y_{n}-\gamma\left(y^{\prime}\right)\right) & \text { for } \quad \gamma\left(y^{\prime}\right) \leq y_{n} \leq \gamma\left(y^{\prime}\right)+t^{\frac{1}{2}} \\
I^{-}\left(y^{\prime}, y_{n}\right)=-t+t^{\frac{1}{2}}\left(y_{n}-\gamma\left(y^{\prime}\right)\right) & \text { for } \quad \gamma\left(y^{\prime}\right) \leq y_{n} \leq \gamma\left(y^{\prime}\right)+t^{\frac{1}{2}} \\
I^{ \pm}\left(y^{\prime}, y_{n}\right)=0 & \text { for } \quad y_{n}>\gamma\left(y^{\prime}\right)+t^{\frac{1}{2}} .
\end{array}
$$

We observe that the functions $I^{ \pm}$are Lipschitz continuous on $Q(x, r) \cap \bar{\Omega}$; hence they belong to $W^{1, \infty}(\Omega)$ and we have:

$$
\left|D_{n} I^{ \pm}\right|=\left\{\begin{array}{lll}
0, & \text { a.e. in } & y_{n}>\gamma\left(y^{\prime}\right)+t^{\frac{1}{2}} \\
t^{\frac{1}{2}}, & \text { a.e. in } & \gamma\left(y^{\prime}\right)<y_{n}<\gamma\left(y^{\prime}\right)+t^{\frac{1}{2}},
\end{array}\right.
$$

while, for $j=1, \ldots, n-1$, we have:

$$
\left|D_{j} I^{ \pm}\right|=\left\{\begin{array}{lll}
0, & \text { a.e. in } & y_{n}>\gamma\left(y^{\prime}\right)+t^{\frac{1}{2}} \\
t^{\frac{1}{2}}\left|D_{j} \gamma\right|, & \text { a.e. in } & \gamma\left(y^{\prime}\right)<y_{n}<\gamma\left(y^{\prime}\right)+t^{\frac{1}{2}} .
\end{array}\right.
$$

Formulas (2.33), (2.34), (2.35) and (2.36) imply that there exists $M_{1}>0$ depending on $\partial \Omega$ (through the $L^{\infty}$-norm of $D \gamma$ ) but independent of $t$ such that

$$
\left|D I^{ \pm}\right| \leq M_{1} t^{\frac{1}{2}} \quad \text { a.e. in } \quad Q(x, r) \cap \bar{\Omega} .
$$

In addition, setting

$$
\Omega_{t}(x) \doteq\left\{y=\left(y^{\prime}, y_{n}\right): y_{n} \geq \gamma\left(y^{\prime}\right)+t^{\frac{1}{2}}\right\} \cap Q(x, r),
$$

we have

$$
\text { meas }\left(Q(x, r) \cap\left(\Omega \backslash \Omega_{t}(x)\right)\right) \rightarrow 0 \quad \text { as } \quad t \rightarrow 0+
$$

and

$$
\operatorname{dist}\left(\Omega_{t}, \partial \Omega \cap Q(x, r)\right) \rightarrow 0 \quad \text { as } \quad t \rightarrow 0+.
$$

Define the map $z: Q(x, r) \cap \bar{\Omega} \rightarrow \mathbf{R}$ by setting

$$
z(y) \doteq \min \left\{v(y), I^{+}(y)\right\} .
$$

Recall (2.33) and (2.34): since $I^{+}(y)=0$ for $y \in \Omega_{t}(x)$, we have

$$
z(y) \leq 0 \quad \forall y \in \Omega_{t}(x) .
$$

Since $I^{+}(y)=t$ and, recalling (2.30), $v(y) \leq t$ for $y \in \partial \Omega$, we have

$$
z(y)=v(y) \quad \forall y \in \partial \Omega \cap Q
$$

while (2.31) implies that

$$
z(x)=0 \quad \forall x \in Z .
$$

Define the map $w: Q(x, r) \cap \bar{\Omega} \rightarrow \mathbf{R}$ by setting

$$
w(y) \doteq \max \left\{z(y), I^{-}(y)\right\} .
$$

By (2.42) and since $I^{-}(y)=0$ for $y \in \Omega_{t}(x)$, we have

$$
w(y)=0 \quad \forall y \in \Omega_{t}(x) .
$$


Since $I^{-}(y)=-t$ and, by (2.30) and (2.43), $z(y)=v(y) \geq-t$ for $y \in \partial \Omega \cap Q$, we have

$$
w(y)=v(y) \quad \forall y \in \partial \Omega \cap Q .
$$

Putting together (2.44), (2.45), (2.46), (2.47), we obtain

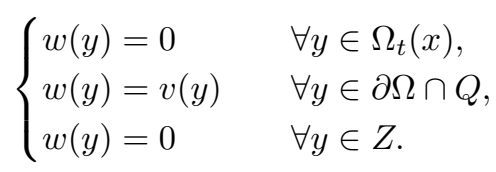

By Stampacchia's theorem we have that $w$ belongs to $W^{1, \infty}(\Omega \cap Q)$ and from formulas (2.41), (2.45) and (2.48) we have

$$
D w(x) \in\left\{D I^{-}(x), D I^{+}(x), D v(x)\right\} \text { for a.e. } x \in \Omega \cap Q .
$$

Step 3. In order to globalize the construction let $\left\{x^{j}, j=1, \ldots, m\right\}$ be a family of points in $\partial \Omega$ and let $\left\{Q_{j}=Q\left(x^{j}, r_{j}\right), j=1, \ldots, m\right\}$ be a corresponding covering of $\partial \Omega$ by cubes for which the previous conditions hold. Take an open subset $\Omega_{0}$ compactly contained in $\Omega$ such that

$$
\Omega \subseteq\left(\bigcup_{j=1}^{m} Q_{j}\right) \cup \Omega_{0}
$$

and a partition of unity $\left\{\phi_{j}, j=1, \ldots, m\right\}$ of $\partial \Omega$ associated to the family $\left\{Q_{j}\right\}$. For each $j \in\{1 \ldots, m\}$ set $v_{j} \doteq \phi_{j} v, Z_{j}=\left(Q_{j} \cap \bar{\Omega}\right) \backslash \operatorname{supp}\left(\phi_{j} v\right)$ and, assuming that $t$ is small enough so that the equivalent of condition (2.33) is satisfied for every $j \in\{1 \ldots, m\}$, define, as in the previous step, sets $\Omega_{t}\left(x^{j}\right)$, maps $I_{j}^{ \pm}$and maps $w_{j} \in W^{1, \infty}\left(Q_{j} \cap \Omega\right)$ with the following properties (see (2.48) and (2.49)):

$$
\begin{aligned}
& w_{j}=0 \quad \text { in } \Omega_{t}\left(x^{j}\right), \\
& w_{j}=0 \quad \text { in } Z_{j}, \\
& w_{j}=v_{j} \quad \text { in } \partial \Omega \cap Q_{j}, \\
& D w_{j}(x) \in\left\{I_{j}^{+}(x), I_{j}^{-}(x), D v_{j}(x)\right\} \quad \text { for a.e. } x \in \Omega \cap Q_{j},
\end{aligned}
$$

where

$$
\left|D I_{j}^{ \pm}\right| \leq M_{2} t^{\frac{1}{2}} \text { a.e. in } Q_{j} \cap \bar{\Omega}, \forall j=1, \ldots, m
$$

for a suitable $M_{2}>0$ independent on $t$, with the condition (see (2.38) and (2.39)):

$$
\text { meas }\left(Q_{j} \cap\left(\Omega \backslash \Omega_{t}\left(x^{j}\right)\right)\right) \rightarrow 0 \quad \text { as } \quad t \rightarrow 0+\quad \forall j \in\{1 \ldots, m\} .
$$

Now define

$$
\Omega_{t} \doteq \bigcup_{j=1}^{m} \Omega_{t}\left(x_{j}\right)
$$

and

$$
w_{t}(y) \doteq \begin{cases}0, & y \in \Omega_{0}, \\ \sum_{j=1}^{m} w_{j}(y), & y \in\left(\bigcup_{j=1}^{m} Q_{j}\right) \cap \Omega .\end{cases}
$$

We obtain easily (2.24), (2.25) and (2.26) while conditions (2.50) and (2.51) imply that the definition of $w_{t}$ is well posed and that $w_{t} \in W^{1, \infty}(\Omega)$; (2.52) ensures that 
(2.27) is satisfied. In order to prove (2.29) observe that we have

$$
D w_{t}(y)= \begin{cases}0, & y \in \Omega_{0}, \\ \sum_{j=1}^{m} D w_{j}(y), & y \in\left(\bigcup_{j=1}^{m} Q_{j}\right) \cap \Omega .\end{cases}
$$

Considering only the nonzero values of $D w_{t}$ and recalling (2.53), we have

$$
\begin{aligned}
D w_{t}(x) & =\sum_{j=1}^{m} D w_{j}(x) \\
& =\sum_{j \in A} D I_{j}^{+}(x)+\sum_{j \in B} D I_{j}^{-}(x)+\sum_{j \in C}\left(D \phi_{j}(x) v(x)+\phi_{j}(x) D v(x)\right),
\end{aligned}
$$

where $A, B, C$ are the subsets of $\{1, \ldots, m\}$ on which the $D w_{j}$ assume, respectively, the values $I_{j}^{+}(x), I_{j}^{-}(x), D v_{j}(x)$. Recalling (2.54) and (2.32), and remarking that the computation is performed on points whose distance from $\partial \Omega$ is less than $t^{\frac{1}{2}}$, we have

$$
\left|\sum_{j \in A} D I_{j}^{+}(x)+\sum_{j \in B} D I_{j}^{+}(x)+\sum_{j \in C} D \phi_{j}(x) v(x)\right| \leq M_{3} t^{\frac{1}{2}}+M_{4} t,
$$

where $M_{3}$ and $M_{4}$ are suitable positive constants depending on $M$, on $\partial \Omega$ and on the partition of unity but independent of $u, v$ and $t$. On the other hand, we have

$$
\sum_{j \in C} \phi_{j}(x) D v(x) \in \operatorname{co}(\{0, D v(x)\}) \quad \text { for a.e. } \quad x \in \Omega .
$$

Putting together (2.55), (2.56), (2.57) and (2.58), we obtain (2.29).

Property (2.28) follows easily from (2.26), (2.27), (2.29) and from the fact that the distance of $\Omega_{t}$ from $\partial \Omega$ goes to zero as $t \rightarrow 0+($ see (2.40) $)$.

Step 4. To end the proof we are left to remove the assumption $u \equiv 0$. Set $\tilde{v} \doteq v-u$, $\tilde{u} \equiv 0$ and define a map $\tilde{w}_{t}$ as in step 3 relative to the functions $\tilde{u}$ and $\tilde{v}$; then set

$$
w_{t} \doteq u+\tilde{w}_{t} \text {. }
$$

Properties (2.24)-(2.29) can be easily verified.

\section{EXISTENCE OF A VISCOSITY SOLUTION BY MAXIMALITY}

In this section we consider the Dirichlet problem for the Hamilton-Jacobi equation

$$
\mathcal{P}_{\varphi}: \begin{cases}F(x, u, D u)=0 & \text { in } \Omega \\ u=\varphi & \text { in } \partial \Omega\end{cases}
$$

and suggest a method for finding a viscosity solution based on the maximization of the integral. Consider a continuous function $F: \Omega \times \mathbb{R} \times \mathbb{R}^{n} \rightarrow \mathbb{R}$ and its lower convex envelope with respect to the last variable: $F^{* *}: \Omega \times \mathbb{R} \times \mathbb{R}^{n} \rightarrow \mathbf{R}$. Given an element $\varphi \in W^{1, \infty}(\Omega)$ we consider the problem of finding a map $u \in \varphi+W_{0}^{1, \infty}(\Omega)$ such that

$$
F(x, u(x), D u(x))=0
$$

in the viscosity sense. Since we deal with locally Lipschitz continuous functions, our solution turns out to solve equation (3.1) almost everywhere. We introduce also the auxiliary relaxed equation

$$
F^{* *}(x, u(x), D u(x))=0,
$$


the corresponding Dirichlet problem

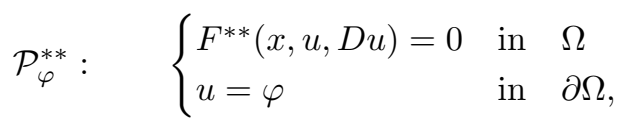

and impose the following

Hypothesis H. Let $F: \Omega \times \mathbb{R} \times \mathbb{R}^{n} \rightarrow \mathbb{R}$ be continuous. We assume that $F^{* *}: \Omega \times \mathbb{R} \times \mathbb{R}^{n} \rightarrow \mathbb{R}$ is continuous, that

$$
\lim _{|\xi| \rightarrow \infty} F(x, u, \xi)=+\infty \quad \text { uniformly in }(x, u) \in \Omega \times \mathbb{R}
$$

and that

$$
\begin{aligned}
\left\{(x, u, \xi) \in \Omega \times \mathbb{R} \times \mathbb{R}^{n}: F(x, u, \xi)>0\right\} & \\
& =\left\{(x, u, \xi) \in \Omega \times \mathbb{R} \times \mathbb{R}^{n}: F^{* *}(x, u, \xi)>0\right\} .
\end{aligned}
$$

We give also the

Definition 2. Given a map $\varphi \in W^{1, \infty}(\Omega)$, we set

$$
S_{\varphi} \doteq\left\{u \in \varphi+W_{0}^{1, \infty}(\Omega): F^{* *}(x, u(x), D u(x)) \leq 0 \text { for a.e. } x \in \Omega\right\} .
$$

Theorem 1. Assume hypothesis $H$ and let $\varphi \in W^{1, \infty}(\Omega)$ be such that $S_{\varphi}$ is nonempty. Then there exists a unique map $\bar{u} \in S_{\varphi}$ such that

$$
\bar{u}(x) \geq u(x) \quad \forall x \in \Omega, \quad \forall u \in S_{\varphi} .
$$

Proof. Step 1. $S_{\varphi}$ is sequentially compact with respect to the weak* convergence in $W^{1, \infty}(\Omega)$.

In order to prove this assertion take a sequence $\left(v_{k}\right)$ in $S_{\varphi}$. By condition (3.3), $\left(v_{k}\right)$ is bounded in $W^{1, \infty}(\Omega)$; hence there exists a subsequence (still denoted by $\left.\left(v_{k}\right)\right)$ such that $v_{k} \stackrel{*}{\rightarrow} w$ in $W^{1, \infty}(\Omega)$. Clearly $w \in \varphi+W_{0}^{1, \infty}(\Omega, \mathbf{R})$ and we have to prove that

$$
F^{* *}(x, w(x), D w(x)) \leq 0 \quad \text { for a.e. } x \in \Omega .
$$

Take a test function $\theta \in C_{c}^{\infty}(\Omega, \mathbf{R})$ and consider the functional

$$
S_{\varphi} \ni v \mapsto \int_{\Omega} \theta(x) F^{* *}(x, v(x), D v(x)) d x .
$$

By the convexity of $F^{* *}$ with respect to $D v$, the functional turns out to be lower semicontinuous with respect to weak* convergence in $W^{1, \infty}(\Omega)$ and then we have

$$
\begin{array}{r}
\int_{\Omega} \theta(x) F^{* *}(x, w(x), D w(x)) d x \\
\leq \liminf _{k \rightarrow \infty} \int_{\Omega} \theta(x) F^{* *}\left(x, v_{k}(x), D v_{k}(x)\right) d x \leq 0 .
\end{array}
$$

By the arbitrariness of $\theta$ we obtain (3.7).

Step 2. Consider the functional

$$
S_{\varphi} \ni v \mapsto \int_{\Omega} v(x) d x .
$$


The Sobolev-Rellich embedding theorem implies that it is continuous with respect to the weak* convergence in $W^{1, \infty}(\Omega)$; by Step 1 and by the Weierstrass Theorem we conclude that it is bounded on $S_{\varphi}$ and that there exists $\bar{u} \in S_{\varphi}$ such that

$$
\int_{\Omega} \bar{u} d x \geq \int_{\Omega} u d x \quad \forall u \in S_{\varphi} .
$$

Step 3. To prove pointwise maximality of $\bar{u}$ take an arbitrary $v \in S_{\varphi}$ and assume, by contradiction, that the open set $E \subseteq \Omega$ on which $v>\bar{u}$ is nonempty. Then define

$$
w \doteq \bar{u} \wedge v=\max \{\bar{u}, v\}
$$

We have that

$$
w(x)=v(x) \quad \forall x \in E, \quad w(x)=\bar{u}(x) \quad \forall x \in \Omega \backslash E
$$

and, by Stampacchia's theorem,

$$
D w(x)=D v(x) \quad \text { for a.e. } x \in E, \quad D w(x)=D \bar{u}(x) \quad \text { for a.e. } x \in \Omega \backslash E .
$$

By direct inspection we see that $w \in S_{\varphi}$ and that, in addition,

$$
\int_{\Omega} w d x>\int_{\Omega} \bar{u} d x
$$

This contradicts (3.8) and then $\bar{u} \geq v$ in $\Omega$. The uniqueness of $\bar{u}$ is immediate.

Theorem 2. Assume hypothesis $H$ and let $\varphi \in W^{1, \infty}(\Omega)$ be such that $S_{\varphi}$ is nonempty. Then the map $\bar{u}$ defined in Theorem 1 is a viscosity solution of $\mathcal{P}_{\varphi}$ and of $\mathcal{P}_{\varphi}^{* *}$.

Proof. Step 1. We start by proving the following.

Claim. Given a function $u$ in $S_{\varphi}$ and an open set $\Lambda \Subset \Omega$, there exists a sequence $\left(u_{k}\right)$ in $C^{\infty}(\Lambda)$ and a sequence $\left(\epsilon_{k}\right)$ in $\mathbb{R}^{+}$such that

$$
\begin{gathered}
u_{k} \longrightarrow u \quad \text { uniformly on } \Lambda, \\
\epsilon_{k} \longrightarrow 0 \quad \text { as } \quad k \rightarrow \infty, \\
F^{* *}\left(x, u_{k}(x), D u_{k}(x)\right) \leq \epsilon_{k} \quad \forall x \in \Lambda \quad \forall k \in \mathbb{N} .
\end{gathered}
$$

Take a regularizing sequence $\left(\rho_{k}\right)$ and set, for $k$ sufficiently large,

$$
u_{k}(x) \doteq\left(\rho_{k} * u\right)(x), \quad x \in \Lambda .
$$

We have, for $x \in \Lambda$,

$$
\begin{aligned}
F^{* *}\left(x, u_{k}(x), D u_{k}(x)\right)= & {\left[F^{* *}\left(x, u_{k}(x), D u_{k}(x)\right)-F^{* *}\left(x, u(x), D u_{k}(x)\right)\right] } \\
& +F^{* *}\left(x, u(x), D u_{k}(x)\right) .
\end{aligned}
$$

Since $u$ is continuous on $\bar{\Lambda}, u_{k}$ converges uniformly to $u$ on such a set and then, by the uniform boundedness of $\left\|D u_{k}\right\|_{L^{\infty}}$, we have

$$
\left[F^{* *}\left(x, u_{k}(x), D u_{k}(x)\right)-F^{* *}\left(x, u(x), D u_{k}(x)\right)\right] \rightarrow 0 \text { uniformly on } \Lambda .
$$


On the other hand, remarking that the convolution is a convex combination, by the convexity of $\xi \mapsto F^{* *}(x, u, \xi)$ and by the Jensen inequality, we have, for $x \in \Lambda$ :

$$
\begin{array}{r}
F^{* *}\left(x, u(x), D u_{k}(x)\right)=F^{* *}\left(x, u(x), \rho_{k} * D u(x)\right) \\
=F^{* *}\left(x, u(x), \int_{B\left(x, \frac{1}{k}\right)} \rho_{k}(x-y) D u(y) d y\right) \\
\quad \leq \int_{B\left(x, \frac{1}{k}\right)} \rho_{k}(x-y) F^{* *}(x, u(x), D u(y)) d y \\
=\int_{B\left(x, \frac{1}{k}\right)} \rho_{k}(x-y)\left[F^{* *}(x, u(x), D u(y))-F^{* *}(y, u(y), D u(y))\right] d y \\
\quad+\int_{B\left(x, \frac{1}{k}\right)} \rho_{k}(x-y) F^{* *}(y, u(y), D u(y)) d y
\end{array}
$$

where we assume that $k$ is sufficiently large so that, for a suitable open set $U \Subset \Omega$,

$$
\Lambda+B\left(0, \frac{1}{k}\right) \subset U
$$

By the uniform continuity of $u$ on $\bar{U}$, the term in the square parenthesis in (3.13) goes to zero uniformly as $k \rightarrow \infty$, and, since $u$ belongs to $S_{\varphi}$, the integral in (3.14) is nonpositive. Hence there exists a sequence $\left(\epsilon_{k}^{\prime}\right)$ in $\mathbf{R}^{+}$with $\epsilon_{k}^{\prime} \rightarrow 0$ and $\bar{k} \in \mathbf{I N}$ such that

$$
F^{* *}\left(x, u(x), D u_{k}(x)\right) \leq \epsilon_{k}^{\prime} \quad \forall x \in \Lambda \quad \forall k \geq \bar{k} .
$$

Putting together (3.12) and (3.15) we prove the claim.

Step 2. We prove now that $\bar{u}$ is a viscosity subsolution of the relaxed equation (3.2). Take $x_{0} \in A^{+}(\bar{u}), \xi \in D^{+} \bar{u}\left(x_{0}\right)$ and assume, by contradiction, that

$$
F^{* *}\left(x_{0}, u\left(x_{0}\right), \xi\right)=\delta>0 .
$$

Take an open set $\Lambda \Subset \Omega$ such that $x_{0} \in \Lambda$ and consider the sequence $\left(u_{k}\right)$ of the previous step. By a standard argument (see for example Proposition 2.2 in [2]), recalling the smoothness of $u_{k}$ and point (iv) in Lemma 1, there exists a sequence $\left(x_{k}\right)$ in $\Lambda$ such that

$$
x_{k} \rightarrow x_{0} \quad \text { and } \quad D u_{k}\left(x_{k}\right) \rightarrow \xi \quad \text { as } k \rightarrow \infty .
$$

Hence, by the continuity of $F^{* *}$, by (3.9) and by (3.16), we have

$$
F^{* *}\left(x_{k}, u_{k}\left(x_{k}\right), D u_{k}\left(x_{k}\right)\right) \longrightarrow F^{* *}\left(x_{0}, u\left(x_{0}\right), \xi\right)=\delta>0 \text { as } k \rightarrow \infty .
$$

Inequality (3.17) contradicts (3.10)-(3.11) and then

$$
F^{* *}(x, u(x), \xi) \leq 0 \quad \forall x \in A^{+}(\bar{u}) \quad \text { and } \quad \forall \xi \in D^{+} \bar{u}(x) .
$$

This proves the claim of step 2 .

By the same argument we have that

$$
F^{* *}(x, u(x), \xi) \leq 0 \quad \forall x \in A^{-}(\bar{u}) \quad \text { and } \quad \forall \xi \in D^{-} \bar{u}(x) .
$$

Step 3. We prove now that $\bar{u}$ is a viscosity supersolution of the relaxed equation (3.2). Take $x_{0} \in A^{-}(\bar{u}), \xi \in D^{-} \bar{u}\left(x_{0}\right)$ and assume, by contradiction, that

$$
F^{* *}\left(x_{0}, \bar{u}\left(x_{0}\right), \xi\right)<0 .
$$


By the continuity of $F^{* *}$ and of $\bar{u}$, we infer from (3.19) the existence of $R>0$, $r>0$ and $t>0$ such that $B\left(x_{0}, R\right) \subseteq \Omega$ and

$$
F^{* *}(x, \bar{u}(x)+s, \eta) \leq 0 \quad \forall x \in B\left(x_{0}, R\right), \quad \forall s \in[0, t], \quad \forall \eta \in \overline{B(\xi, r)} .
$$

By Lemma 2 there exist $\rho \in] 0, R[$, a map

$$
\hat{u} \in \bar{u}+W_{0}^{1, \infty}(\Omega)=\varphi+W_{0}^{1, \infty}(\Omega)
$$

and a nonempty open set

$$
\Lambda \subseteq B\left(x_{0}, \rho\right) \subseteq B\left(x_{0}, R\right)
$$

with the following properties:

$$
\begin{aligned}
& \hat{u}(x)=\bar{u}(x) \quad \text { for a.e. } \quad x \in \Omega \backslash \Lambda, \\
& \bar{u}(x) \leq \hat{v}(x) \leq \bar{u}(x)+t \quad \text { for a.e. } x \in \Lambda, \\
& D \hat{u}(x)=D \bar{u}(x) \quad \text { for a.e. } x \in \Omega \backslash \Lambda, \\
& |D \hat{u}(x)-D \bar{u}(x)|=r \quad \text { for a.e. } x \in \Lambda, \\
& \hat{u}(x)>\bar{u}(x) \quad \forall x \in \Lambda .
\end{aligned}
$$

Conditions (3.22)-(3.26), together with (3.20), ensure that

$$
F^{* *}(x, \hat{u}(x), D \hat{u}(x)) \leq 0 \text { for a.e. } x \in \Omega \text {. }
$$

Recalling (3.21) and Definition 2, inequality (3.28) implies that $\hat{u}$ is an element of $S_{\varphi}$. Hence inequality (3.27) contradicts the maximality of $\bar{u}$ (formula (3.6) in Theorem 11) and then we have that

$$
F^{* *}(x, \bar{u}(x), \xi) \geq 0 \quad \forall x \in A^{-}(\bar{u}) \quad \text { and } \quad \forall \xi \in D^{-} \bar{u}(x) .
$$

This proves the claim of step 3 and, in particular, putting together (3.18) and (3.29) we have, actually, that

$$
F^{* *}(x, \bar{u}(x), \xi)=0 \quad \forall x \in A^{-}(\bar{u}) \quad \text { and } \quad \forall \xi \in D^{-} \bar{u}(x) .
$$

Step 2 and step 3 imply that $\bar{u}$ is a viscosity solution of $\mathcal{P}_{\varphi}^{* *}$.

Step 4. Let us consider now the nonconvex equation (3.1).

Take $x_{0} \in A^{+}(\bar{u}), \xi \in D^{+} \bar{u}\left(x_{0}\right)$ and assume, by contradiction, that

$$
F\left(x_{0}, \bar{u}\left(x_{0}\right), \xi\right)>0 .
$$

Recalling (3.4) in hypothesis $\mathrm{H}$ we have also $F^{* *}\left(x_{0}, \bar{u}\left(x_{0}\right), \xi\right)>0$ and then, invoking step 2, we obtain a contradiction.

Take $x_{0} \in A^{-}(\bar{u}), \xi \in D^{-} \bar{u}\left(x_{0}\right)$ and assume, by contradiction, that

$$
F\left(x_{0}, \bar{u}\left(x_{0}\right), \xi\right)<0 .
$$

Since, by definition, $F^{* *} \leq F$, we have $F^{* *}\left(x_{0}, \bar{u}\left(x_{0}\right), \xi\right)<0$ and then, invoking step 3 , we obtain a contradiction also in this case.

Hence $\bar{u}$ is a viscosity solution of $\mathcal{P}_{\varphi}$ and this ends the proof.

We end this section with a brief discussion on the existence result that we have proved. First of all we exhibit a function $F$ satisfying our hypotheses.

Example 1. Let $g: \Omega \times \mathbb{R} \rightarrow \mathbb{R}$ be a continuous function such that $g(x, u) \geq \delta>0$ $\forall(x, u) \in \Omega \times \mathbb{R}$. Set

$$
F(x, u, \xi) \doteq g(x, u)\left(\left(|\xi|^{2}-1\right)^{2}-4\right), \quad(x, u, \xi) \in \Omega \times \mathbb{R} \times \mathbb{R}^{n} .
$$

It is easy to see that $F$ satisfies hypothesis $\mathrm{H}$ and that Theorem 2 applies for every $\varphi \in W^{1, \infty}(\Omega)$ such that $|D \varphi| \leq \sqrt{3}$ a.e. in $\Omega$. 
Remark 3. We discuss briefly hypothesis $\mathrm{H}$ showing that, in general, it cannot be weakened. First of all notice that condition (3.3) can be replaced by the almost equivalent following one:

$$
\exists R>0, \delta>0: \quad F(x, u, \xi) \geq \delta \quad \forall(x, u, \xi) \in \Omega \times \mathbb{R} \times \mathbb{R}^{n}, \quad|\xi| \geq R .
$$

If this property fails, in general, we cannot expect existence of solutions for the problem $\mathcal{P}_{\varphi}$, as is trivially shown, for example, by the one dimensional case corresponding to $\Omega=] 0,1[, \varphi \equiv 0, F(\xi)=\xi-1$.

If condition (3.4) in hypothesis $\mathrm{H}$ is dropped, then problem $\mathcal{P}_{\varphi}$ may fail to have a solution, as is shown by the following one dimensional example.

Example 2. Let $F: \mathbb{R} \rightarrow \mathbb{R}$ be given by

$$
F(\xi) \doteq \begin{cases}2|\xi+1|-1 & \text { for } \xi<0 \\ 1 & \text { for } 0 \leq \xi<3 \\ \frac{1}{2}(\xi-1) & \text { for } \xi \geq 3,\end{cases}
$$

so that we have

$$
F^{* *}(\xi) \doteq \begin{cases}-2 \xi-3 & \text { for } \xi<-1 \\ \frac{1}{2}(\xi-1) & \text { for } \xi \geq-1 .\end{cases}
$$

Condition (3.4) is violated since $F(0)=1$ while $F^{* *}(0)=-\frac{1}{2}$.

Consider the problems

$$
\left\{\begin{array}{l}
\left.F\left(u^{\prime}(t)\right)=0 \quad \text { in }\right] 0,1[ \\
u(0)=u(1)=0
\end{array}\right.
$$

and

$$
\left\{\begin{array}{l}
\left.F^{* *}\left(u^{\prime}(t)\right)=0 \quad \text { in }\right] 0,1[ \\
u(0)=u(1)=0 .
\end{array}\right.
$$

We have $\{\xi \in \mathbb{R}: F(\xi)=0\}=\left\{-\frac{3}{2},-\frac{1}{2}\right\}$ and $\left\{\xi \in \mathbb{R}: F^{* *}(\xi)=0\right\}=\left\{-\frac{3}{2}, 1\right\}$. It is easy to see that the convex problem associated to $F^{* *}$ admits infinitely many solutions and that, in particular, the maximal one is given by the formula

$$
\bar{u}(t)= \begin{cases}t & \text { for } 0 \leq t<\frac{3}{5} \\ -\frac{3}{2}(t-1) & \text { for } \frac{3}{5} \leq t \leq 1 .\end{cases}
$$

On the other hand the nonconvex problem associated to $F$ has no solutions since $u(0)=0$ and $u^{\prime}(t) \in\left\{-\frac{3}{2},-\frac{1}{2}\right\}$ a.e. in $] 0,1\left[\right.$ imply $u(1) \leq-\frac{1}{2}$.

\section{Continuous DePendence on Boundary Data}

The viscosity solution $\bar{u}$ of $\mathcal{P}_{\varphi}$ and of $\mathcal{P}_{\varphi}^{* *}$ provided by Theorem 2 is unique, in the sense that if two elements of $S_{\varphi}$ are pointwise greater than any other element of the set, then they necessarily coincide. Hence we may investigate if $\bar{u}$ depends continuously on the boundary datum with respect to some topology and in this section we face this problem. Since we have obtained the maximal solution of the nonconvex problem as a solution of the convex one, keeping the notation of the previous section, we assume, without loss of generality, that $F=F^{* *}$. 
Theorem 3. Let $\Omega$ be an open bounded subset of $\mathbf{R}^{n}$ with Lipschitz boundary $\partial \Omega$. Let $F: \Omega \times \mathbb{R} \times \mathbb{R}^{n} \rightarrow \mathbf{R}$ be a continuous function, convex in the last variable, satisfying (3.3). Let $\left(\varphi_{k}\right), k \in \mathbf{I N}_{0}$, be a bounded sequence in $W^{1, \infty}(\Omega)$ such that

$$
F\left(x, \varphi_{k}(x), D \varphi_{k}(x)\right) \leq 0 \quad \text { for a.e. } x \in \Omega \quad \forall k \in \mathbb{N}_{0}
$$

and assume that

$$
\varphi_{k} \longrightarrow \varphi_{0} \quad \text { uniformly on } \partial \Omega \quad \text { as } \quad k \rightarrow \infty \text {. }
$$

For every $k \in \mathbb{N}_{0}$ consider the problem

$$
\mathcal{P}_{\varphi_{k}}: \quad \begin{cases}F(x, u, D u)=0 & \text { in } \Omega, \\ u=\varphi_{k} & \text { in } \partial \Omega,\end{cases}
$$

and let $\bar{u}_{k}$ be the viscosity solution of $\mathcal{P}_{\varphi_{k}}$ given by Theorem 2 , Then

$$
\bar{u}_{k} \stackrel{*}{\rightarrow} \bar{u}_{0} \quad \text { in } W^{1, \infty}(\Omega) .
$$

Proof. Step 1. For every $\epsilon \geq 0$ and for every $k \in \mathbb{N}_{0}$, introduce the set

$$
S_{\varphi_{k}}^{\epsilon} \doteq\left\{u \in \varphi_{k}+W_{0}^{1, \infty}(\Omega): F(x, u(x), D u(x)) \leq \epsilon \text { for a.e. } x \in \Omega\right\},
$$

remarking that $S_{\varphi_{k}}^{0}=S_{\varphi_{k}}$ (see (3.5) in Definition 2). By the same argument used in Theorem 1 for every $\epsilon>0$, we may infer the existence of a map $\bar{u}_{k}^{\epsilon}$ such that

$$
\bar{u}_{k}^{\epsilon} \geq u \quad \text { in } \quad \Omega \quad \forall u \in S_{\varphi_{k}}^{\epsilon} .
$$

Fix $k \in \mathbb{I}_{0}$; by (3.3) in hypothesis $\mathrm{H}$ there exists a positive constant $M$ such that

$$
\left.\left\|\bar{u}_{k}^{\epsilon}\right\|_{W^{1, \infty}(\Omega)} \leq M \quad \forall \epsilon \in\right] 0,1[.
$$

Take any sequence $\left(\epsilon_{j}\right)$ with $\epsilon_{j} \rightarrow 0+$ such that the sequence $\left(u_{k}^{\epsilon_{j}}\right)$ converges weakly* in $W^{1, \infty}(\Omega)$ (and uniformly by Rellich's theorem) and call $v$ its limit. Take a test function $\theta \in C_{c}^{\infty}(\Omega)$ with $\int_{\Omega} \theta d x=1$, and observe that, by the convexity of $\xi \mapsto F(x, u, \xi)$, we have:

$$
\begin{aligned}
& \int_{\Omega} F(x, v(x), D v(x)) \theta(x) d x \\
& \leq \liminf _{j \rightarrow \infty} \int_{\Omega} F\left(x, \bar{u}_{k}^{\epsilon_{j}}(x), D \bar{u}_{k}^{\epsilon_{j}}(x)\right) \theta(x) d x \leq \epsilon_{j} \rightarrow 0 .
\end{aligned}
$$

By the arbitrariness of $\theta$ we have

$$
F(x, v(x), D v(x)) \leq 0 \quad \text { for a.e. } \quad x \in \Omega
$$

and, since $v$ belongs to $\varphi_{k}+W_{0}^{1, \infty}(\Omega)$, being the uniform limit of a sequence of elements of this set, it follows that

$$
v \in S_{\varphi_{k}} .
$$

On the other hand we have $S_{\varphi_{k}} \subseteq S_{\varphi_{k}}^{\epsilon}$ for every $\epsilon>0$ and, consequently,

$$
\int_{\Omega} \bar{u}_{k} d x \leq \int_{\Omega} \bar{u}_{k}^{\epsilon} d x \quad \forall \epsilon>0 .
$$

Inequality (4.3) and the uniform convergence $\bar{u}_{k}^{\epsilon_{j}} \rightarrow v$ as $j \rightarrow \infty$ imply that

$$
\int_{\Omega} \bar{u}_{k} d x \leq \int_{\Omega} v d x
$$


Recalling (4.2) and that, by definition, $\bar{u}_{k}$ is the maximal element of $S_{\varphi_{k}}$, (4.4) implies that $v=\bar{u}_{k}$. By the arbitrariness of the subsequence $\left(\bar{u}_{k}^{\epsilon_{j}}\right)$ we conclude that

$$
\bar{u}_{k}^{\epsilon} \stackrel{*}{\rightarrow} \bar{u}_{k} \quad \text { in } \quad W^{1, \infty}(\Omega) \quad \text { as } \quad \epsilon \rightarrow 0+.
$$

Step 2.

Claim. For every $\epsilon \in] 0,1\left[\right.$ there exists a sequence $\left(u_{k}\right)$ in $S_{\varphi_{k}}^{\epsilon}$ such that

$$
u_{k} \longrightarrow \bar{u}_{0} \quad \text { strongly in } W^{1,1}(\Omega) .
$$

Since $\left.\bar{u}_{0}\right|_{\partial \Omega}=\left.\varphi_{0}\right|_{\partial \Omega}$ and $\varphi_{k} \rightarrow \varphi_{0}$ uniformly on $\partial \Omega$, remarking that all functions are uniformly bounded in $W^{1, \infty}(\Omega)$, we may fix $k \in \mathbb{I}$ and apply Lemmaß with $u, v$ replaced by the functions $\bar{u}_{0}$ and $\varphi_{k}$, obtaining sets $\Omega_{k} \subseteq \Omega$, a sequence $\sigma_{k} \rightarrow 0+$ and maps $u_{k} \in W^{1, \infty}(\Omega)$ such that

$$
\begin{aligned}
& \Omega_{k} \subseteq \Omega_{k+1} \quad \forall k \in \mathbb{N} ; \\
& \text { meas }\left(\Omega \backslash \Omega_{k}\right) \rightarrow 0 \quad \text { as } \quad k \rightarrow \infty \\
& u_{k}=\bar{u}_{0} \text { in } \Omega_{k} ; \\
& u_{k}=\varphi_{k} \text { in } \partial \Omega ; \\
& \left|u_{k}-\bar{u}_{0}\right|,\left|u_{k}-\varphi_{k}\right| \leq \sigma_{k} \quad \text { in } \Omega \backslash \Omega_{k} ; \\
& \operatorname{dist}\left(D u_{k}(x), \operatorname{co}\left(\left\{D \bar{u}_{0}(x), D \varphi_{k}(x)\right\}\right)\right) \leq \sigma_{k} \quad \text { for a.e. } \quad x \in \Omega .
\end{aligned}
$$

Formula (4.12) implies that the sequence $\left(u_{k}\right)$ is bounded in $W^{1, \infty}(\Omega)$; hence (4.7), (4.8) and (4.9) imply (4.6). Since (4.10) implies that $u_{k}$ belongs to $\varphi_{k}+W^{1, \infty}(\Omega)$, we have only to prove that there exists $k_{\epsilon} \in \mathbb{N}$ such that $u_{k} \in S_{\varphi_{k}}^{\epsilon}$ for every $k \geq k_{\epsilon}$.

By construction we have $u_{k}=\bar{u}_{0}$ and $D u_{k}=D \bar{u}_{0}$ a.e. in $\Omega_{k}$; hence

$$
F\left(x, u_{k}(x), D u_{k}(x)\right) \leq 0 \text { for a.e. } \quad x \in \Omega_{k} .
$$

In order to perform the computation on $\Omega \backslash \Omega_{k}$ we observe that by (4.12) there exist measurable functions

$$
\lambda_{k}: \Omega \rightarrow[0,1]
$$

and

$$
\theta_{k}: \Omega \rightarrow \mathbb{R}^{n}: \quad\left|\theta_{k}(x)\right| \leq \sigma_{k} \quad \text { for a.e. } \quad x \in \Omega \backslash \Omega_{k},
$$

such that

$$
D u_{k}(x)=\lambda_{k}(x) D \bar{u}_{0}(x)+\left(1-\lambda_{k}(x)\right) D \varphi_{k}(x)+\theta_{k}(x) \quad \text { for a.e. } \quad x \in \Omega \backslash \Omega_{k} .
$$

Hence we have

$$
\begin{aligned}
& F\left(x, u_{k}, D u_{k}\right)=F\left(x, u_{k}, \lambda_{k} D \bar{u}_{0}+\left(1-\lambda_{k}\right) D \varphi_{k}+\theta_{k}\right) \\
& =\left[F\left(x, u_{k}, \lambda_{k} D \bar{u}_{0}+\left(1-\lambda_{k}\right) D \varphi_{k}+\theta_{k}\right)\right. \\
& \left.-F\left(x, u_{k}, \lambda_{k} D \bar{u}_{0}+\left(1-\lambda_{k}\right) D \varphi_{k}\right)\right] \\
& +F\left(x, u_{k}, \lambda_{k} D \bar{u}_{0}+\left(1-\lambda_{k}\right) D \varphi_{k}\right) .
\end{aligned}
$$

The square parentheses in (4.15) can be estimated by observing that, by (4.14), the arguments are close to each other in the uniform topology; hence, by the continuity 
of $F$, there exists $k_{\epsilon}^{\prime} \in \mathbb{I N}$ such that

$$
\begin{aligned}
& \mid F\left(x, u_{k}, \lambda_{k} D \bar{u}_{0}+\left(1-\lambda_{k}\right) D \varphi_{k}+\theta_{k}\right) \\
& \quad-F\left(x, u_{k}, \lambda_{k} D \bar{u}_{0}+\left(1-\lambda_{k}\right) D \varphi_{k}\right) \mid \leq \frac{\epsilon}{3} \quad \text { a.e. in } \quad \Omega \backslash \Omega_{k},
\end{aligned}
$$

for every $k \geq k_{\epsilon}^{\prime}$. Then we have

$$
F\left(x, u_{k}, \lambda_{k} D \bar{u}_{0}+\left(1-\lambda_{k}\right) D \varphi_{k}\right) \leq \lambda_{k} F\left(x, u_{k}, D \bar{u}_{0}\right)+\left(1-\lambda_{k}\right) F\left(x, u_{k}, D \varphi_{k}\right)
$$

and

$$
\begin{aligned}
& F\left(x, u_{k}, D \bar{u}_{0}\right)=[\left.F\left(x, u_{k}, D \bar{u}_{0}\right)-F\left(x, \bar{u}_{0}, D \bar{u}_{0}\right)\right] \\
&+F\left(x, \bar{u}_{0}, D \bar{u}_{0}\right), \\
& F\left(x, u_{k}, D \varphi_{k}\right)=\left[\begin{array}{c}
\left.F\left(x, u_{k}, D \varphi_{k}\right)-F\left(x, \varphi_{k}, D \varphi_{k}\right)\right] \\
+ \\
+F\left(x, \varphi_{k}, D \varphi_{k}\right) .
\end{array}\right.
\end{aligned}
$$

The square parentheses in (4.17) and in (4.18) can be estimated by recalling that we are performing the computation on the set $\Omega \backslash \Omega_{k}$ on which, recalling (4.11), the function $u_{k}$ is close to $\bar{u}_{0}$ and to $\varphi_{k}$ in the uniform topology. Hence, by the continuity of $F$, there exists $k_{\epsilon}^{\prime \prime} \in \mathbb{I}$ such that

$$
\left|F\left(x, u_{k}, D \bar{u}_{0}\right)-F\left(x, \bar{u}_{0}, D \bar{u}_{0}\right)\right| \leq \frac{\epsilon}{3} \quad \text { a.e. in } \quad \Omega \backslash \Omega_{k}
$$

and

$$
\left|F\left(x, u_{k}, D \varphi_{k}\right)-F\left(x, \varphi_{k}, D \varphi_{k}\right)\right| \leq \frac{\epsilon}{3} \quad \text { a.e. in } \quad \Omega \backslash \Omega_{k},
$$

for every $k \geq k_{\epsilon}^{\prime \prime}$. Recalling that

$$
F\left(x, \bar{u}_{0}, D \bar{u}_{0}\right) \leq 0, \quad F\left(x, \varphi_{k}, D \varphi_{k}\right) \leq 0 \quad \text { a.e. on } \Omega,
$$

and inserting (4.16), (4.17), (4.18), (4.19), (4.20) and (4.21) in (4.15), we have

$$
F\left(x, u_{k}, D u_{k}\right) \leq \epsilon \quad \text { a.e. in } \quad \Omega \backslash \Omega_{k} \quad \forall k \geq \max \left(k_{\epsilon}^{\prime}, k_{\epsilon}^{\prime \prime}\right) .
$$

This last inequality, together with (4.13), concludes the proof of the claim.

Step 3. Consider the sequence $\left(\bar{u}_{k}\right)$ which, by (3.3) of hypothesis $\mathrm{H}$, is bounded in $W^{1, \infty}(\Omega)$. Take any weakly* converging subsequence, still denoted by $\left(\bar{u}_{k}\right)$, and call $v$ its weak* limit in $W^{1, \infty}(\Omega)$.

Claim. The map $v$ belongs to $S_{\varphi}$.

Clearly $v \in \varphi+W_{0}^{1, \infty}(\Omega)$, since it is the weak* limit of a sequence whose elements coincide at the boundary with the elements of the sequence $\left(\varphi_{k}\right)$ which converges uniformly to $\varphi$ on $\partial \Omega$. As in step 1 take a test function $\theta \in C_{c}^{\infty}(\Omega)$ and observe that, by the convexity of $\xi \mapsto F(x, u, \xi)$, we have:

$$
\begin{array}{r}
\int_{\Omega} F(x, v(x), D v(x)) \theta(x) d x \\
\leq \liminf _{k \rightarrow \infty} \int_{\Omega} F\left(x, \bar{u}_{k}(x), D \bar{u}_{k}(x)\right) \theta(x) d x \leq 0,
\end{array}
$$

since $\bar{u}_{k} \in S_{\varphi_{k}}$ for every $k$. By the arbitrariness of $\theta$ we have

$$
F(x, v(x), D v(x)) \leq 0 \quad \text { for a.e. } \quad x \in \Omega .
$$

Hence the claim is proved and, in particular, we have:

$$
v \leq \bar{u}_{0} \quad \text { in } \Omega \text {. }
$$


Claim. $v=\bar{u}_{0}$.

Fix an index $k$ and recall (4.5). Since $\bar{u}_{k} \stackrel{*}{\rightarrow} v$ in $W^{1, \infty}(\Omega)$ as $k \rightarrow \infty$, by a diagonal argument we may find a sequence $\epsilon_{k} \rightarrow 0+$ such that

$$
\bar{u}_{k}^{\epsilon_{k}} \stackrel{*}{\rightarrow} v \quad \text { in } \quad W^{1, \infty}(\Omega) \quad \text { as } \quad k \rightarrow \infty .
$$

By the result of step 2 we may construct a sequence $\left(u_{k}\right)$ such that $u_{k} \in S_{\varphi_{k}}^{\epsilon_{k}} \quad \forall k \in$ $\mathbf{N}$ and $u_{k} \rightarrow \bar{u}_{0}$ uniformly and strongly in $W^{1,1}(\Omega)$. Hence we have

$$
\int_{\Omega} u_{k} d x \longrightarrow \int_{\Omega} \bar{u}_{0} d x \quad \text { as } \quad k \rightarrow \infty
$$

In addition, by the definition (4.1) of $\bar{u}_{k}^{\epsilon}$, we have

$$
\int_{\Omega} u_{k} d x \leq \int_{\Omega} \bar{u}_{k}^{\epsilon_{k}} d x \quad \forall k \in \mathbf{N} .
$$

Then, collecting (4.23), (4.24) and (4.25), we conclude that

$$
\int_{\Omega} \bar{u}_{0} d x=\lim _{k \rightarrow \infty} \int_{\Omega} u_{k} d x \leq \lim _{k \rightarrow \infty} \int_{\Omega} \bar{u}_{k}^{\epsilon_{k}} d x=\int_{\Omega} v d x .
$$

Putting together (4.22) and (4.26) we obtain that $v=\bar{u}_{0}$ and, by the arbitrariness of the chosen subsequence, it follows that the whole sequence $\left(\bar{u}_{k}\right)$ converges weakly* to $\bar{u}_{0}$ in $W^{1, \infty}(\Omega)$.

This ends the proof of the theorem.

Remark 4. In general we cannot expect continuous dependence in any strong Sobolev topology, as is shown by the following.

Example 3. Let $n=2, \Omega=] 0,1[\times] 0,1\left[\right.$ and $F: \mathbb{R}^{2} \rightarrow \mathbb{R}$ be given by

$$
F(\xi)=F\left(\xi_{1}, \xi_{2}\right)=\max \left\{\left(|\xi|^{2}-1\right),\left|\xi_{2}\right|\right\} .
$$

Observe that hypothesis $\mathrm{H}$ is satisfied and that $\left\{\left(\xi_{1}, \xi_{2}\right): F\left(\xi_{1}, \xi_{2}\right)=0\right\}=\left\{\left(\xi_{1}, \xi_{2}\right)\right.$ : $\left.\xi_{1} \in[-1,1], \xi_{2}=0\right\}$. Consider the sequence of boundary data given by

$$
\varphi_{k}\left(x_{1}, x_{2}\right)=\frac{1}{k} \sin \left(k x_{1}\right), \quad k \in \mathbb{I N}, \quad \varphi_{0} \equiv 0 .
$$

We have

$$
D \varphi_{k}\left(x_{1}, x_{2}\right)=\left(\cos \left(k x_{1}\right), 0\right), \quad k \in \mathbb{I}, \quad D \varphi_{0}=0
$$

and, clearly,

$$
\varphi_{k} \stackrel{*}{\rightarrow} \varphi_{0} \quad \text { in } \quad W^{1, \infty}(\Omega) .
$$

For every $k \in \mathbb{N}, \varphi_{k}$ is the unique solution of the problem

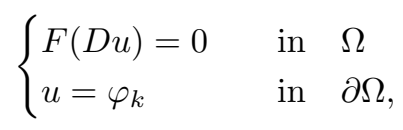

and, being smooth, it is also a viscosity solution. Indeed let $u \in \varphi_{k}+W_{0}^{1, \infty}(\Omega)$ be such that $F(D u)=0$ a.e. in $\Omega$. We have, necessarily, $D_{2}\left(u-\varphi_{k}\right)=0$ a.e. in $\Omega$ and then, by Poincaré's inequality, $\left(u-\varphi_{k}\right) \equiv 0$ in $\Omega$. Hence $\varphi_{k}$ is the maximal solution of the problem and the sequence $\left(\varphi_{k}\right)$ does not converge strongly to $\varphi_{0}$ in any Sobolev space. 
In this example we have exhibited a function $F$ such that the set of extremal points of the closure of the convex hull $\overline{\mathrm{co}}(E)$ of the set $E$ in which $F$ vanishes is strictly contained in $\overline{\mathrm{co}}(E)$. In Theorem 5 below we will show that if $E$ coincides with the set of extremal points of $\overline{\mathrm{co}}(E)$, then we have continuous dependence in the strong topology of $W^{1, p}$ for $1 \leq p<\infty$.

\section{The homogeneous CASE}

In this section we discuss briefly some consequences of our results in the homogeneous case corresponding to a continuous function $F: \mathbb{R}^{n} \rightarrow \mathbf{R}$ and to the problem

$$
\mathcal{P}_{\varphi}^{0}: \quad\left\{\begin{array}{lll}
F(D u)=0 & \text { in } & \Omega \\
u=\varphi & \text { in } & \partial \Omega .
\end{array}\right.
$$

We recall from [9] the following.

Theorem 4. Let $\Omega$ be an open bounded convex subset of $\mathbb{R}^{n}$ and let $F: \mathbb{R}^{n} \rightarrow \mathbf{I R}$ be a convex function such that $F(\xi) \rightarrow \infty$ as $|\xi| \rightarrow \infty$ and $F(0)=0$. Set

$$
E \doteq\left\{\xi \in \mathbb{R}^{n}: F(\xi)=0\right\}
$$

and assume that $E=\partial(\mathrm{co} E)$. Then the problem

$$
\left\{\begin{array}{lll}
F(D u)=0 & \text { in } & \Omega \\
u=0 & \text { in } & \partial \Omega
\end{array}\right.
$$

admits a unique viscosity solution.

In the quoted paper, the solution is given by an explicit formula, based on the gauge function of the set $E$ for the unique viscosity solution which turns out to be Lipschitz continuous and that the authors call fundamental. Clearly the maximal solution provided by Theorem 2 in section 3 of this paper coincides with the fundamental one and then we may say that, in a certain sense, the notion of maximal solution generalizes the notion of fundamental solution.

We see now a case in which we may improve Theorem 3, obtaining continuous dependence on boundary data in a stronger topology.

Theorem 5. Let $\Omega$ be an open bounded subset of $\mathbf{I R}^{n}$ with Lipschitz boundary $\partial \Omega$ and let $F: \mathbb{R}^{n} \rightarrow \mathbb{R}$ be a continuous function such that $F(\xi) \rightarrow \infty$ as $|\xi| \rightarrow \infty$ and $\left\{\xi \in \mathbb{R}^{n}: F(\xi)>0\right\}=\left\{\xi \in \mathbb{R}^{n}: F^{* *}(\xi)>0\right\}$. Set

$$
E \doteq\left\{\xi \in \mathbb{R}^{n}: F^{* *}(\xi)=0\right\}
$$

and assume

$$
E=\operatorname{extr}(\overline{\mathrm{co}}(E)) .
$$

Let $\left(\varphi_{k}\right), k \in \mathbb{N}_{0}$, be a bounded sequence in $W^{1, \infty}(\Omega)$ such that

$$
F^{* *}\left(D \varphi_{k}\right) \leq 0 \quad \text { a.e. in } \Omega \quad \forall k \in \mathbb{N}
$$

and

$$
\varphi_{k} \longrightarrow \varphi_{0} \quad \text { uniformly on } \partial \Omega \quad \text { as } \quad k \rightarrow \infty \text {. }
$$

For every $k \in \mathbb{I N}_{0}$ call $\bar{u}_{k}$ the maximal solution of problem $\mathcal{P}_{\varphi_{k}}^{0}$ given by Theorem 2. Then

$$
\bar{u}_{k} \longrightarrow \bar{u}_{0} \quad \text { strongly in } \quad W^{1, p}(\Omega) \quad \text { as } \quad k \rightarrow \infty \quad \forall p \in[1, \infty[.
$$


Proof. By Theorem 3 we know that $\bar{u}_{k} \stackrel{*}{\rightarrow} \bar{u}_{0}$ in $W^{1, \infty}(\Omega)$. Since, for every $k \in \mathbf{I N}_{0}$, $\bar{u}_{k}$ solves the equation in $\mathcal{P}_{\varphi_{k}}^{0}$ almost everywhere in $\Omega$, by (5.1), we have

$$
D \bar{u}_{0}(x) \in \operatorname{extr}\left(\overline{\operatorname{co}}\left\{D \bar{u}_{k}(x), k \in \mathbf{I N}_{0}\right\}\right) \quad \text { for a.e. } \quad x \in \Omega .
$$

Hence, by Theorem 1 in [14, we have

$$
D \bar{u}_{k}(x) \longrightarrow D \bar{u}_{0}(x) \text { for a.e. } x \in \Omega \text {. }
$$

This ends the proof.

Example 4. Consider the function $F$ given in example 1 with $g \equiv 1$. It is easy to see that the hypotheses of Theorem 5 are satisfied for any sequence such that $\left|D \varphi_{k}\right| \leq \sqrt{3}$ a.e. in $\Omega$.

\section{REFERENCES}

[1] G. Barles, Solutions de viscosité des équations de Hamilton-Jacobi, Mathématiques et Applications, 17, Springer-Verlag, Berlin, 1994. MR1613876 (2000b:49054)

[2] M. Bardi, I. Capuzzo Dolcetta, Optimal control and viscosity solutions of Hamilton-JacobiBellmann equations, Birkhäuser, Basel, 1997. MR1484411 (99e:49001)

[3] A. Bressan, F. Flores, On total differential inclusions. Rend. Sem. Mat. Uni. Padova 92 (1994), 9-16. MR.1320474 (96b:35244)

[4] F. Camilli, A. Siconolfi, Maximal solutions for a class of degenerate Hamilton-Jacobi problems, Indiana Univ. Math. J., 48 (1999), 1111-1131. MR.1736967(2001a:49028)

[5] I. Capuzzo Dolcetta, P.L. Lions, Viscosity solutions of Hamilton-Jacobi equations and state constraints problems, Trans. Amer. Math. Soc., 318 (1990), 643-683. MR951880 (90g:49021)

[6] P. Cardaliaguet, B. Dacorogna, W. Gangbo, N. Georgy, Geometric restrictions for the existence of viscosity solutions, Annales Henri Poincaré Anal. Non Linéaire, 16 (1999), 189-220. MR.1674769 (99k:35023)

[7] M.G. Crandall, L.C. Evans, P.L. Lions, Some properties of viscosity solutions of HamiltonJacobi equations, Trans. Amer. Math. Soc. 282 (1984), 487-502. MR732102 (86a:35031)

[8] B. Dacorogna, P. Marcellini, General existence theorems for Hamilton-Jacobi equations in scalar and vectorial cases, Acta Math. 178 (1997), 1-37. MR1448710 (98d:35029)

[9] B. Dacorogna, P. Marcellini, Viscosity solutions, almost everywhere solutions and explicit formulas, Trans. Amer. Math. Soc. 356 (2004), 4643-4653. MR2067137(2005c:49064)

[10] B. Dacorogna, P. Marcellini, Implicit partial differential equations, Birkhäuser, Basel, 1999. MR1702252 (2000f:35005)

[11] F.S. De Blasi, G. Pianigiani, On the Dirichlet problem for Hamilton-Jacobi equations. A Baire category approach, NoDEA Nonlinear Differential Equations Appl. 6 (1999), 13-34. MR.1674778 (2000d:35021)

[12] L.C. Evans, R.G. Gariepy, Measure theory and fine properties of functions, CRC Press, Boca Raton (1992). MR:1158660 (93f:28001)

[13] P.L. Lions, Generalized solutions of Hamilton-Jacobi equations, Research Notes in Math. 69, Pitman, London, 1982. MR667669 (84a:49038)

[14] A. Visintin, Strong convergence results related to strict convexity, Comm. Partial Diff. Equations 9(5) (1984), 439-466. MR741216 (85f:49028)

[15] S. Zagatti, On the minimum problem for nonconvex scalar functionals, SIAM J. Math. Analysis 37/3 (2005), 982-995. MR2191784(2006j:49004)

[16] S. Zagatti, Solutions of vectorial Hamilton-Jacobi equations and minimizers of nonquasiconvex functionals, J. Math. Anal. Appl. 335 (2007), 1143-1160. MR2346897

[17] S. Zagatti, Uniqueness and continuous dependence on boundary data for integro-extremal minimizers of the functional of the gradient, J. Convex Analysis 14 (2007), 705-727. MR2350812

[18] S. Zagatti, Minimizers of non convex scalar functionals and viscosity solutions of HamiltonJacobi equations, Calc. Var. and PDE's 31 (2008), 511-519. MR2372904

Department of Mathematics, Scuola Internazionale Superiore di Studi Avanzati, Via Beirut, 2/4, I-34014 Trieste, Italy 